\title{
Origins of water in the Solar System leading to habitable worlds
}

\author{
Karen Meech \\ Institute for Astronomy, Honolulu, Hawaii, USA \\ email: meech@ifa.hawaii.edu
}

\begin{abstract}
Life on Earth depends on an aqueous biochemistry, and water is a key component of habitability on Earth and for likely other habitable environments in the solar system. While water is ubiquitous in the interstellar medium, and plays a key role in protoplanetary disk chemistry, the inner solar system is relatively dry. We now have evidence for potentially thousands of extrasolar planets, dozens of which may be located in their host stars habitable zones. Understanding how planets in the habitable zone accrete their water, is key to understanding the likelihood for habitability. Given that many disk models show that Earth formed inside the water-ice snow line of our solar system, understanding how the inner solar system received its water is important for understanding the potential for other planetary systems to host habitable worlds. Boundaries for the timing of the water delivery are constrained by cosmochemistry and geochemistry. Possible scenarios for the delivery of water to the inner solar system include adsorption on dust from protoplanetary disk gas, chemical reactions on the early earth, and delivery from planetesimals forming outside the water-ice snow line. This talk will set the stage for understanding the isotopic and geochemical markers along with the dynamical delivery mechanisms that will help uncover the origins of Earths water. This introduction will provide an overview for understanding the distribution of water in the solar system, in particular for the inner solar system and terrestrial planets Xand the details will be developed in the subsequent talks. Additionally information will be presented regarding new inner solar system reservoirs of water that can shed light on origins (the main belt comets), and new research about water in the Earth.
\end{abstract}

Keywords. 